\title{
The continuum limit of QED. Renormalization group analysis and the question of triviality
}

\author{
M. Göckeler ${ }^{a, b}$, R. Horsley ${ }^{\text {a,b }}$, E. Laermann ${ }^{b}$, P. Rakow ${ }^{c, 1}$, G. Schierholz ${ }^{\text {b,c }}$, R. Sommer ${ }^{\text {d }}$ and \\ U.-J. Wiese ${ }^{b}$ \\ a Institut für Theoretische Physik, RWTH Aachen, Sommerfeldstraße, W-5100 Aachen, FRG \\ b Gruppe Theorie der Elementarteilchen, Höchstleistungsrechenzentrum HLRZ, c/o KFA, Postfach 1913, W-5170 Jülich, FRG \\ c Deutsches Elektronen-Synchrotron DESY, Notkestraße 85, W-2000 Hamburg 52, FRG \\ d Fachbereich Physik, Gesamthochschule Wuppertal, Gaußstraße 20, W-5600 Wuppertal, FRG
}

Received 21 July 1990

\begin{abstract}
Recently there has been much interest in the chiral phase transition of QED and its implication for the continuum limit. In this letter we present the first lattice calculation of the renormalized charge and fermion mass. We use these results to derive the Callan-Symanzik $\beta$-function. We find that the renormalized charge vanishes at the critical point, which indicates triviality. By examining different renormalization group trajectories we address the issue of whether QED can be viewed as a consistent cut-off theory.
\end{abstract}

\section{Introduction}

In this Letter we shall continue our efforts [1] to understand the ultraviolet behavior of QED using lattice techniques. We are particularly interested in knowing whether QED has a non-trivial continuum limit. This is one of the most fundamental questions in field theory, which recently has also received considerable interest by various authors attempting to construct microscopic models of dynamical symmetry breaking [2].

Kogut, Dagotto and Kocic [3] have discovered that non-compact QED undergoes a chiral phase transition at strong coupling. This result was confirmed by Booth, Kenway and Pendleton [4] and us [1]. Recently, it has been shown rigorously [5] that chiral symmetry is broken spontaneously in the strong coupling limit. It is agreed that the phase transition is of second order. This means that non-compact QED possesses a continuum limit. The problem then re-

Address after September 1: Institut für Theoretische Physik, WE2, Freie Universität Berlin, Arnimallee 14, W-1000 Berlin, Germany. duces to finding out whether the Callan-Symanzik $\beta$ function has an ultraviolet stable zero.

In our previous paper [1] we have studied the critical exponents of the chiral phase transition. The results turned out to be consistent with the predictions of a gaussian model, which we regarded as a first indication that QED is non-interacting in the continuum limit. In this paper we shall concentrate on the renormalized charge and the renormalized fermion mass in the vicinity of the critical point. It is the evolution of the renormalized charge as one moves towards the critical point at fixed bare charge which determines the Callan-Symanzik $\beta$-function.

The lattice version of non-compact QED using Kogut-Susskind fermions is given by the action

$$
\begin{aligned}
& S=S_{\mathrm{G}}+S_{\mathrm{F}} \\
& S_{\mathrm{G}}=\frac{1}{2} \beta \sum_{x, \mu<\nu} F_{\mu \nu}^{2}(x) \\
& S_{\mathrm{F}}=\sum_{x}\left(\frac { 1 } { 2 } \sum _ { \mu } ( - 1 ) ^ { x _ { 1 } + \ldots + x _ { \mu - 1 } } \left(\bar{\chi}_{x} \mathrm{e}^{\mathrm{i} A \mu(x)} \chi_{x+\mu}\right.\right. \\
& \left.\left.\quad-\bar{\chi}_{x+\mu} \mathrm{e}^{-\mathrm{i} A_{\mu}(x)} \chi_{x}\right)+m \bar{\chi}_{x} \chi_{x}\right)
\end{aligned}
$$


where $F_{\mu \nu}(x)=A_{\mu}(x)+A_{\nu}(x+\mu)-A_{\mu}(x+\nu)$ $-A_{\nu}(x)$. The lattice constant has been set equal to 1 for convenience, and $\beta=1 / e^{2}$, where $e$ is the bare charge. The reason for taking Kogut-Susskind fermions is that $S_{\mathrm{F}}$ preserves a chiral $U(1) \times U(1)$ symmetry for finite lattice spacing at $\mathrm{m}=0$. In the classical continuum limit $S_{\mathrm{F}}$ describes four degenerate Dirac fermions (flavors).

We have performed calculations on $8^{4}, 8^{3} \times 16$ and $12^{4}$ lattices at $\beta=0.16,0.17,0.18,0.19,0.20,0.21$, 0.22 and $m=0.02,0.04,0.09,0.16$. At $\beta=0.16$ and 0.22 our runs on the $12^{4}$ lattice have not been completed yet. Therefore we will use the numbers obtained on the $8^{4}$ lattice in these cases. For generating the gauge field configurations we have used the (exact) hybrid Monte Carlo algorithm [6]. Details of the performance of the algorithm for QED can be found in ref. [1]. We have chosen periodic boundary conditions for the gauge fields and periodic (anti-periodic) spatial (temporal) boundary conditions for the Grassmann fields. On the $12^{4}$ and $8^{3} \times 16$ lattices we have accumulated $O(300)$ gauge field configurations, each separated by $5-10$ trajectories, for each value of $\beta$ and $m$, while on the $8^{4}$ lattices our sample consists of $O(100)$ configurations.

\section{The renormalized charge and fermion mass}

The renormalized charge, $e_{\mathrm{R}}$ is related to the bare charge by

$\epsilon_{\mathrm{R}}^{2}=Z_{3} e^{2}$,

Where $Z_{3}$ is the wave function renormalization constant of the photon. The latter can be defined by [7]

$Z_{3}=\lim _{k \rightarrow 0} D(k)$,

where

$D(k)=\frac{1}{3} \beta \sum_{x, \mu<\nu} \mathrm{e}^{\mathrm{i} k x}\left\langle F_{\mu \nu}(x) F_{\mu \nu}(0)\right\rangle$.

We cannot compute $D(0)$ on a periodic lattice because of the boundary conditions, which means that $D(k)$ has to be extrapolated to $k=0$ appropriately.

It has turned out that the photon field $A_{\mu}$ fluctuates strongly. Therefore we have computed $D(k)$ from the identity
$\mathrm{i}-D\left(k^{(\mu)}\right)=\sum_{x} \mathrm{e}^{\mathrm{i} k^{(\mu)} x}\left\langle A_{\mu}(x) j_{\mu}(0)\right\rangle$,

which follows from the equation of motion [7], and which suffers less from these fluctuations. Here, $j_{\mu}$ is the electromagnetic current, and $k^{(\mu)}$ is any momentum with $k_{\mu}^{(\mu)}=0$. The electromagnetic current can be efficiently calculated by means of a stochastic estimator.

In fig. 1 we show $1-D(k)$ as a function of $k^{2}$ on the $12^{4}$ lattice at $\beta=0.21$ and $m=0.04$. This figure is typical of what we obtain for light renormalized fermion masses. For larger masses $1-D(k)$ is essentially constant. The extrapolation of $D(k)$ to $k=0$ can reliably be done by fitting a smooth function to the data. The reason is that the $k^{2}$ dependence of $D(k)$ is determined by the magnitude of the renormalized fermion mass, which on our larger lattices will always be larger than the smallest fermion lattice momentum. We shall return to the extrapolation in detail in section 3 .

In order to determine the renormalized fermion mass, $m_{R}$, we have studied the fermion propagator. Since this is not gauge invariant, we have to fix the gauge. We have chosen the Landau gauge

$\sum_{\mu}\left[A_{\mu}(x+\mu)-A_{\mu}(x)\right]=0$,

which can be implemented exactly. However, this condition does not eliminate all gauge degrees of freedom in the fermion propagator. Adding an integer multiple of $2 \pi / L_{\mu}$, where $L_{\mu}$ is the extent of the lattice in the $\mu$-direction, to $A_{\mu}(x)$ for all $x$ leaves the action invariant. For the fermions this amounts to a gauge transformation, because they couple to the (compact) link variables. Hence, we can add multiples of $2 \pi / L_{\mu}$ to $A_{\mu}(x)$ so that

$-\frac{\pi}{L_{\mu}}<\bar{A}_{\mu} \leqslant \frac{\pi}{L_{\mu}}, \quad \bar{A}_{\mu}=\frac{1}{V} \sum_{x} A_{\mu}(x)$,

for all $\mu$, where $V$ is the lattice volume. Since in our simulations $\bar{A}_{\mu}$, restricted to the interval $\left(-\pi / L_{\mu}, \pi\right)$ $L_{\mu}$ ), does not give 0 when averaged over the ensemble because of the limited statistics, special care has to be taken. Indeed, $\bar{A}_{\mu}$ changes very little from configuration to configuration.

When computing the Kogut-Susskind fermion propagator, we divide the lattice into elementary hy- 


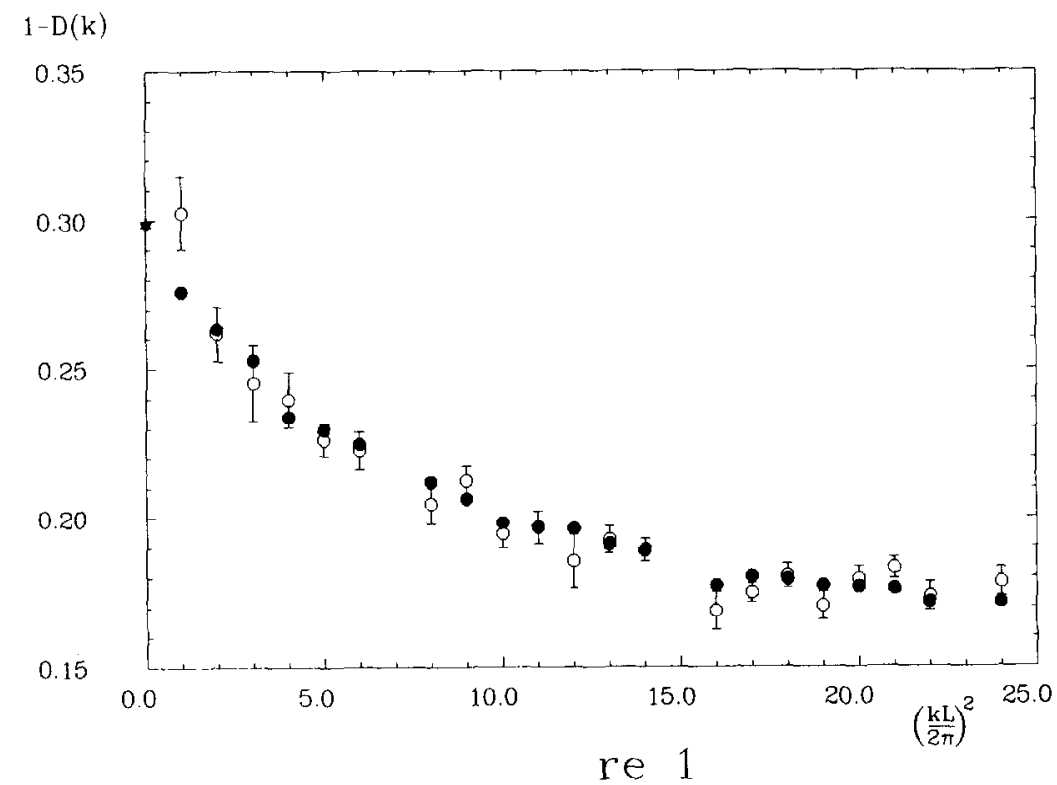

Fig. 1. The current-photon correlation function $1-D(k)$ as a function of $k^{2}$ on the $12^{4}$ lattice at $\beta=0.21$ and $m=0.04$. The open circles are our data. The solid circles are a fit with the 1-loop lattice result. The star is the extrapolation to zero momentum and infinite volume, which gives us the renormalized charge. The error on the latter quantity is smaller than the symbol.

percubes, because the action is invariant under translations of 2 lattice spacings. We compute the propagator

$G_{\omega}(t)=\sum_{x}\langle\chi(2 x+\omega) \tilde{\chi}(0)\rangle$,

where $x$ labels the hypercube, $\omega$ is a 4-vector with components 0 or 1 and $t=2 x_{4}+\omega_{4}$. To extract $m_{\mathrm{R}}$, we shall fit $G_{\omega}(t)$ with the lattice fermion propagator in the presence of a constant background field $B_{\mu} \# 1$. In the analysis we restricted ourselves to $\omega=0$. For $B_{\mu}=0$ this is the only non-vanishing component of the propagator. For $-\pi / L_{\mu}<B_{\mu} \leqslant \pi / L_{\mu}$ the lattice fermion propagator of mass $m_{\mathrm{R}}$ reads

$$
\begin{aligned}
& G_{0}\left(2 x_{4}\right)=\frac{m_{\mathrm{R}}}{L_{4}} \sum_{p_{4}} \mathrm{e}^{2 \mathrm{i} p_{4} x_{4}} \\
& \times\left(\sin ^{2}\left(p_{4}+B_{4}\right)+\sum_{i=1}^{3} \sin ^{2} B_{i}+m_{\mathrm{R}}^{2}\right)^{-1},
\end{aligned}
$$

\#1 Attempts to fit eq. (2.7) with the free propagator $\left(B_{\mu}=0\right)$ did not work. In particular, we found nonvanishing imaginary parts for $G_{0}(t)$, whereas the free propagator is real.

$$
\begin{aligned}
& G_{0}\left(2 x_{4}+1\right)=-\frac{1}{2 m_{\mathrm{R}}} \\
& \quad \times\left[\mathrm{e}^{\mathrm{i} B_{4}} G_{0}\left(2 x_{4}+2\right)-\mathrm{e}^{-\mathrm{i} B_{4}} G_{0}\left(2 x_{4}\right)\right],
\end{aligned}
$$

where

$p_{4}=\frac{2 \pi n}{L_{4}}, \quad n= \pm \frac{1}{2}, \pm \frac{3}{2}, \ldots, \pm \frac{1}{2}\left(L_{4}-1\right)$,

in accord with our choice of anti-periodic temporal boundary conditions. In the fits we allowed for an overall normalization factor as an additional parameter.

In the actual calculations we have computed $G_{\omega}$ for sources distributed over all even points in a given time slice. Subsequently, the average over all time slices is taken. For the analysis we have divided our data in sets of 10-20 configurations. The sets had to be selected such that they do not include jumps of $\bar{A}_{4}$ by $2 \pi / L_{4}$. Then fits starting from $t=1,2$ and 3 (the latter only for our larger lattices ) give consistent results. Furthermore, we find that the fitted values of the background field, $B_{4}$ and $\sum_{i} \sin ^{2} B_{i}$, agree well with $\left\langle\overline{A_{4}}\right\rangle$ and $\sum_{i} \sin ^{2}\left\langle A_{i}\right\rangle$. The mass values obtained from the various sets agree as they should. The errors 


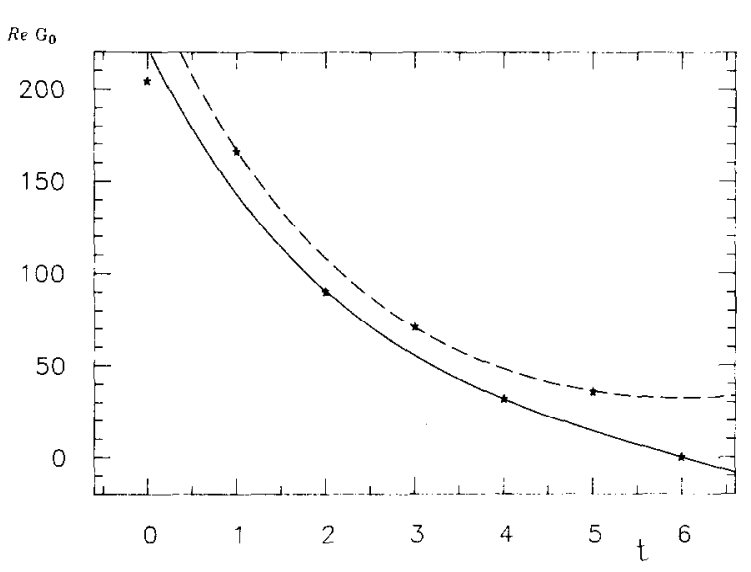

Fig. 2. The real part of the fermion propagator as a function of $t$ on the $12^{4}$ lattice at $\beta=0.21$ and $m=0.04$. The stars are our data. The statistical errors are smaller than the symbols. The curves are a fit to the data for $t \geqslant 1$. Because of the properties of KogutSusskind fermions the even and odd points lie on different curves.

are determined from the fluctuations from set to set. They are typically of the order of a few percent. In fig. 2 we show a correlation function for $\beta=0.21$ and $m=0.04$. Tables of the mass values will be given in a more detailed publication [8].

\section{Renormalization group flow and the $\beta$-function}

To understand the ultraviolet behavior of the theory, we need to know the renormalization group flow with regard to the critical point. The flow is governed by the renormalization group equation

$\left(m_{\mathrm{R}} \frac{\partial e_{\mathrm{R}}^{2}}{\partial m_{\mathrm{R}}}\right)_{\beta \text { fixed }}=\beta\left(e_{\mathrm{R}}^{2}, m_{\mathrm{R}}\right)$,

where $\beta\left(e_{\mathrm{R}}^{2}, m_{\mathrm{R}}\right)$ is the Callan-Symanzik $\beta$-function. Note that $m_{R}$ is given in lattice units and that $m_{R} \rightarrow 0$ at the critical point, i.e. in the continuum limit. The critical coupling was found to be [1] $\beta_{\mathrm{c}} \approx 0.195$, which, according to Lüscher [7] as $Z_{3} \leqslant 1$, leads to the upper bound for the fine-structure constant in the continuum limit:

$\alpha_{\mathrm{R}} \equiv \frac{e_{\mathrm{R}}^{2}}{4 \pi} \leqslant 0.41$.

Since renormalized perturbation theory is expected to be reliable for $\alpha_{\mathrm{R}} \leqslant \pi / 4$ (for 4 flavors), one could argue that the $\beta$-function cannot have a second, ultraviolet stable zero [7]. However, in order to obtain a definite answer, this problem needs to be investigated by non-perturbative methods. This will be done in this section.

The first step is to compute $Z_{3}$ from eqs. (2.2) and (2.4). We have explored several ways of extrapolating $D(k)$ to $k=0$. We shall demonstrate in ref. [8] that the results do not depend on the specific form of the interpolating function. The procedure we find most satisfactory and which we will use in this paper is based on renormalized perturbation theory. Renormalized perturbation theory predicts to 1-loop order

$\frac{1}{e^{2} D(k)}=\frac{1}{e_{\mathrm{R}}^{2}}+\Pi(0, \infty)-\Pi\left(k, L_{\mu}\right)$,

where $\Pi$ derives from the (lattice) vacuum polarization and $e_{\mathrm{R}}^{2}$ refers to the infinite lattice. Since we know $m_{\mathrm{R}}$, the only free parameter in eq. (3.3) is $e_{\mathrm{R}}^{2}$. It turns out that the $k^{2}$ dependence of $D(k)$ can be very well fitted by eq. (3.3). Moreover, we obtain the same values within errors for $e_{\mathrm{R}}^{2}$ on the $8^{4}$ and on the $12^{4}$ lattices. This means that the finite size effects are also accounted for by the perturbative formula. The quality of the fit is shown in fig. 1 for the $12^{4}$ lattice at $\beta=0.21$ and $m=0.04$. The statistical errors on $Z_{3}$ are less than $2 \%$. The actual numbers will be presented in ref. [8].

We have computed the lines of constant renormalized charge in the plane of bare parameters $\beta, m$. Since we know $e_{\mathrm{R}}$ only on the grid of points quoted in section 1, we had to interpolate between them. For the interpolation in $\beta$ and $m$ we have used the formulae $1 / e_{\mathrm{R}}^{2}=a+b \beta$ and $1 / e_{\mathrm{R}}^{2}=c+d \ln m$, respectively. The result is shown by the solid lines in fig. 3 . The uncertainty is about $5 \%$ of the spacing between the trajectories. The most important thing to notice is that when $m$, and so $m_{\mathrm{R}}$, is decreased, $\beta$ must always be decreased to keep $e_{\mathrm{R}}$ constant. This holds on both sides of the critical $\beta$. So the $\beta$-function is positive over the entire range investigated.

In the symmetric phase, $\beta>\beta_{\mathrm{c}}, m_{\mathrm{R}}$ vanishes as $m \rightarrow 0$, and so we expect from a positive $\beta$-function that $e_{\mathrm{R}}=0$ in this limit. In the broken phase, $\beta<\beta_{\mathrm{c}}$, $m_{\mathrm{R}}$ is finite as $m \rightarrow 0$, and so we expect a finite charge renormalization. Therefore all trajectories should end 


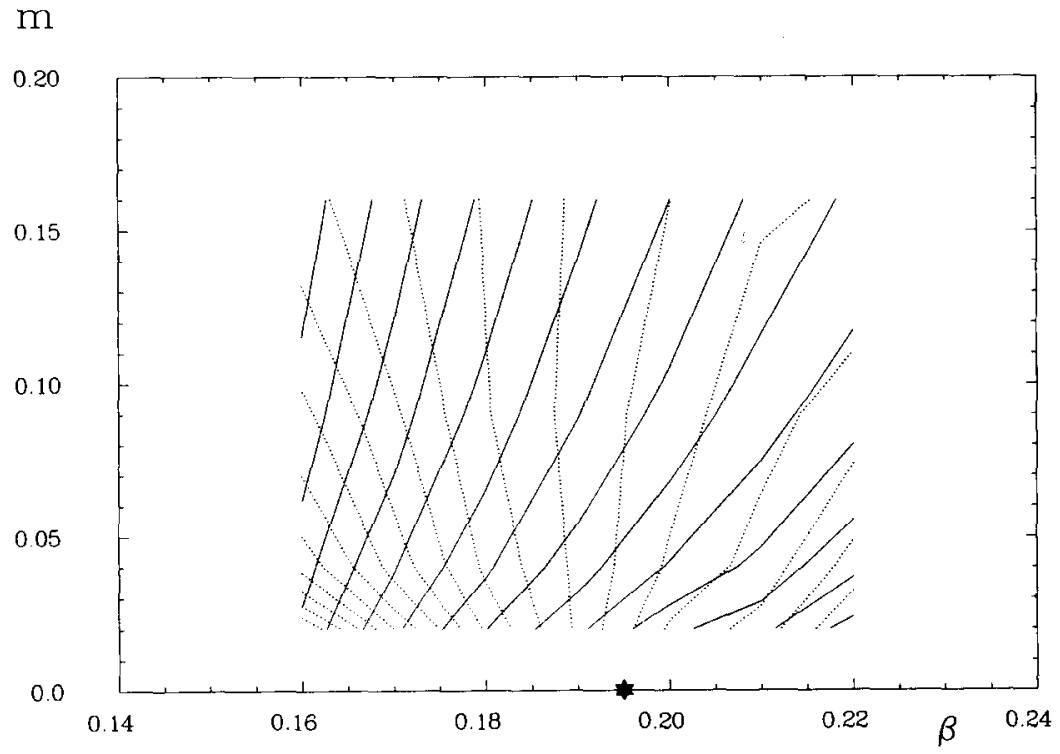

Fig. 3. The renormalization group flow in the region of parameters covered by our calculations. The solid lines are lines of constant renormalized charge, where $e_{\mathrm{R}}^{2}$ ranges from $e_{\mathrm{R}}^{2}=2.8$ (lower right-hand corner) to $e_{\mathrm{R}}^{2}=5.4$ (upper left-hand corner) in steps of 0.2 . The dotted lines are lines of constant $m_{\mathrm{R}} / m_{\mathrm{PS}}$ ranging from 0.4 (lower right-hand corner) to 2.1 (lower left-hand corner) in steps of 0.1 . The uncertainty in the position of the flow lines is about $5 \%$ of the spacing between lines for the $e_{\mathrm{R}}$ lines and about $15 \%$ for the mass-ratio lines. The star indicates the position of the critical point.

at $m=0$ in the broken phase ${ }^{\# 2}$. To make this picture quantitative, we have plotted curves of $1 / e_{\mathrm{R}}^{2}$ against $\ln m_{\mathrm{R}}$ in fig. 4a for various values of $\beta$. The slope of these curves is (see eq. (3.1)) $-\left(1 / e_{\mathrm{R}}^{4}\right) \beta\left(e_{\mathrm{R}}^{2}, m_{\mathrm{R}}\right)$. At lowest order in perturbation theory $\beta\left(e_{\mathrm{R}}^{2}, 0\right)=$ $2 e_{\mathrm{R}}^{4} / 3 \pi^{2}$, which would give a straight line for small $m_{\mathrm{R}}$. Fig. $4 \mathrm{~b}$ shows the $\beta$-function found by differentiating our data compared with the 1-loop $\beta$-function on the infinite lattice. At small $m_{\mathrm{R}}$ the observed $\beta$ function is the same as the 1 -loop $\beta$-function, whereas at larger $m_{\mathrm{R}}$ the observed $\beta$-function is smaller than the 1 -loop result. Note that $\beta\left(e_{\mathrm{R}}^{2}, m_{\mathrm{R}}\right) / e_{\mathrm{R}}^{4}$ is the same for all $\beta$ values, including those in the broken phase. Both these effects can also be seen in fig. $4 a$, where we compare our data with the integrated 1-loop $\beta$ function shifted to coincide with the data point at the smallest value of $m_{\mathrm{R}}$. In the symmetric phase (open

\#2 That this might happen was noted recently by Lüscher [9] and later found in the calculations of Rakow [10]. symbols) the curves extend to $m_{\mathrm{R}}=0$. The renormalization group behavior gives $e_{\mathrm{R}}=0$ in this limit. In particular, $e_{\mathrm{R}}=0$ at the critical point. In the broken phase (solid symbols) the curves end at finite $m_{R}$, and so finite $e_{\mathrm{R}}$. The smaller $m_{\mathrm{R}}$ becomes, the smaller $e_{\mathrm{R}}$ is. For any finite $e_{\mathrm{R}}$ there is a limit on the cutoff using the 1-loop formula (for 4 flavors)

$m_{\mathrm{R}} \geqslant(26 \pm 3) \exp \left(-3 \pi^{2} / 2 e_{\mathrm{R}}^{2}\right)$.

Having found evidence that $e_{\mathrm{R}}=0$ at the critical point, we shall ask now to what extent the theory can be regarded as a consistent cutoff theory. That would require the existence of lines of constant physics, which would not necessarily go to infinite cutoff (correlation length). To answer this question, we need to compare the flow different dimensionless quantities. We have computed the ratio $m_{\mathrm{R}} / m_{\mathrm{PS}}$, where $m_{\mathrm{PS}}$ is the pseudoscalar Goldstone boson mass, on our grid of points and interpolated the result to find the lines of constant mass ratios. In fig. 3 these lines are 


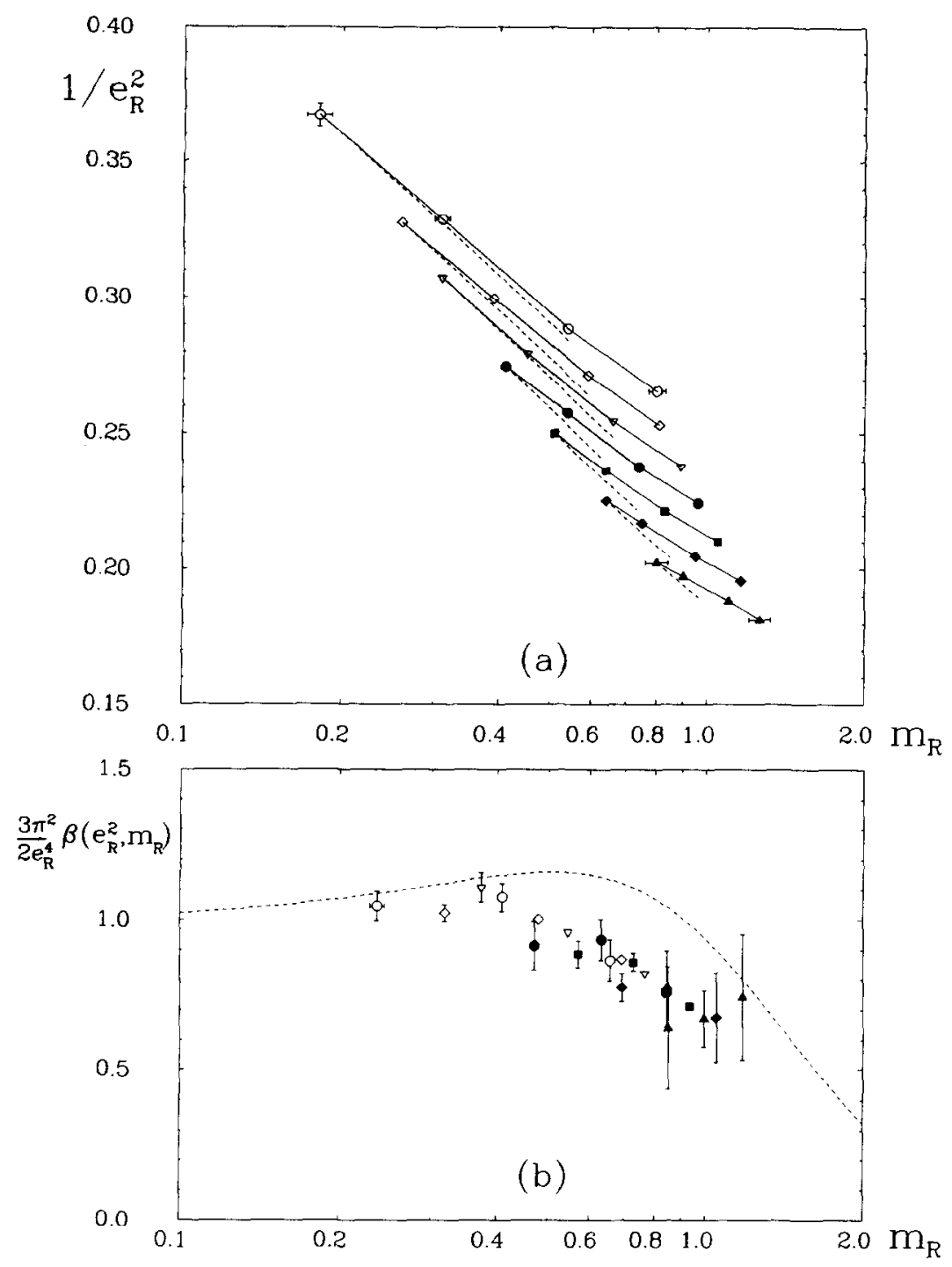

Fig. 4. The $\beta$-function and the relationship between the renormalized charge and the renormalized fermion mass. In (a) we plot $1 / e_{\mathrm{R}}^{2}$ against $m_{\mathbf{R}}$. The symbols refer to the different values of $\beta$ : $\beta=0.16(\Delta), 0.17(\diamond), 0.18(\boldsymbol{\nabla}), 0.19(\bullet), 0.20(\nabla) 0.21(\diamond), 0.22(0)$. The open symbols are for $\beta$ values above $\beta_{\mathrm{c}}$, while the solid symbols are for $\beta$ values below $\beta_{\mathrm{c}}$. The dotted lines are the prediction of the 1 loop lattice $\beta$-function shifted to fit the data point at the smallest value of $m_{\mathrm{R}}$, which corresponds to $m=0.02$. In (b) we show $\beta\left(e_{\mathrm{R}}^{2}\right.$, $\left.m_{\mathrm{R}}\right) /\left(2 e_{\mathrm{R}}^{4} / 3 \pi^{2}\right)$ found by differentiating the data in (a). The symbols are the same as in (a). This is compared with the 1-loop lattice $\beta$-function indicated by the dashed line.

compared with the lines of constant $e_{\mathrm{R}}{ }^{\# 3}$. The two flows are obviously completely different in the parameter range studied. The trajectories of constant mass ratios flow into the critical point in contrast to the lines of constant $e_{\mathrm{R}}$. In perturbation theory $m_{\mathrm{PS}}=m_{\mathrm{R}} f\left(\alpha_{\mathrm{R}}\right)$, and the two sets of trajectories would agree. The inconsistency is most striking for $\beta<\beta_{\mathrm{c}}$,
\#3 For all but two of the grid points $m_{\mathrm{PS}}<2 m_{\mathrm{R}}$, and so the pseudoscalar is a genuine bound state (ignoring the possible decay into two photons). As we go to larger $\beta, m_{\mathrm{R}}$ decreases while the mass of the pseudoscalar produced by our operator [1] increases, and eventually the pseudoscalar becomcs a resonance able to decay into two fermions. There may, of course, be a positronium-like state lighter than $2 m_{R}$, which couples unly weakly to our operator. 
where the $e_{\mathrm{R}}$ trajectories move in the direction of lower $\beta$, while the mass-ratio trajectories move in the direction of larger $\beta$, but it is also clearly present for $\beta>\beta_{\mathrm{c}}$. The correlation length does not have to be very large before the difference between the flows becomes apparent. For example at $e_{\mathrm{R}}^{2}=3.6$ the difference becomes marked when $m_{\mathrm{R}} \lesssim 0.5$ (i.e. at a cutoff which is only two times as large as the fermion mass). When $e_{\mathrm{R}}^{2}$ is smaller, we can reach smaller values of $m_{\mathrm{R}}$ (larger correlation lengths) before this effect is seen. Thus, there are no lines of constant physics in the critical region except possibly for very small values of $e_{\mathrm{R}}^{2}$. This contradicts renormalizability: a change in cutoff cannot be compensated for by a change in the bare parameters. It may be possible to restore renormalizability by adding another interaction to the action. If the extended action is renormalizable, it will have true trajectories of constant physics in the larger space of bare parameters. Various authors have suggested adding a 4-Fermi interaction [11,2].

To illuminate the nature of the chiral transition, we have compared the renormalized fermion mass with the chiral condensate $\langle\bar{\chi} \chi\rangle$ in fig. 5 . We find that $\langle\bar{\chi} \chi\rangle$ and $m_{\mathrm{R}}$ are related in the same way on both sides of $\beta_{\mathrm{c}}$, just as we saw for the $\beta$-function. This suggests that the main effect of the chiral transition is simply to give the fermion a mass, which acts in the same way as the mass induced by $m$ in the symmetric phase. The Miransky model [12] predicts $\langle\bar{\chi} \chi\rangle \propto$ $m_{\mathrm{R}}^{2}$ near the phase transition. We see no sign of any such behavior but find $\langle\bar{\chi} \chi\rangle \propto m_{\mathrm{R}}$ at all $\beta$ values. This indicates that the dimension of $\langle\bar{\chi} \chi\rangle$ is 1 . In fig. 5 we have included for comparison purposes the 1-loop lattice relationship. In agreement with what has been said above, $\langle\bar{\chi} \chi\rangle$ is surprisingly well described by this curve.

\section{Conclusions}

This is the first full investigation of the renormalization of charge in lattice non-compact QED. The key-point in deciding on the triviality of the theory is knowing its interactions in the continuum limit. We were able to simulate the theory at small $e_{\mathrm{R}}$, where we find agreement between the measured $\beta$-function and that predicted by renormalized perturbation theory. This allowed us to extrapolate to the continuum

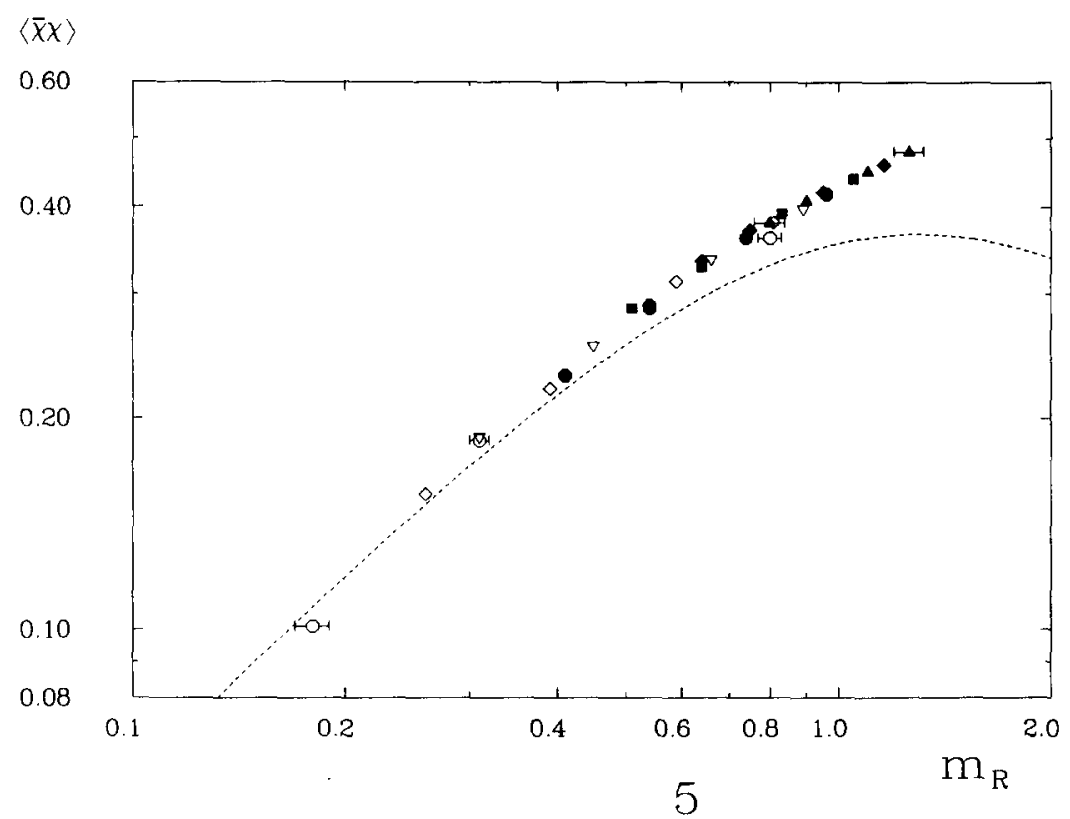

Fig. 5. The chiral condensate against the renormalized fermion mass. The symbols are the same as in fig. 4 . The open symbols are in the chirally symmetric phase, while the solid symbols are in the broken phase. The dashed line is the 1-loop lattice result, which is included for comparison. 
limit by means of the renormalization group equation. We find that $e_{\mathrm{R}}=0$ at the critical point. Our measured $\beta$-function is always positive, whatever side of $\beta_{\mathrm{c}}$ we look.

By comparing the renormalization group trajectories found by keeping $e_{\mathrm{R}}$ constant and by keeping the ratio of fermion mass to pseudoscalar mass, $m_{\mathrm{R}} / m_{\mathrm{PS}}$, constant, we show that there are no lines of constant physics for renormalized couplings as low as $\alpha_{\mathrm{R}} \approx 0.3$ (a value which is supposed to lie inside the domain in which renormalized perturbation theory can be trusted) and quite moderate cutoffs. Our conclusion from this is that pure QED is not renormalizable in this region. This pattern of renormalization group flow was also found from Schwinger-Dyson equations [10].

We have presented evidence that the photon decouples at the critical point. Is it possible that the fermion and the bound states still interact in this limit? The candidate models without photons are NambuJona-Lasinio and Yukawa-type interactions. These are also believed to be trivial theories, because their $\beta$-functions are positive. In any case, the scaling window would be merely the point $\beta=\beta_{\mathrm{c}}$ and $m=0$.

\section{Acknowledgement}

We would like to thank $M$. Lüscher for helpful conversations. The numerical calculations have been mostly performed at the HLRZ with some results on the $8^{3} \times 16$ lattices at the RHRK Kaiserlautern. We thank both institutions for their support.

\section{References}

[ 1 ] M. Göckeler, R. Horsley, E. Lacrmann, P. Rakow, G. Schierholz, R. Sommer and U.-J. Wiese, Nucl. Phys. B 334 (1990) 527.

[2] B. Holdom, Phys. Rev. D 24 (1981) 1441;

K. Yamawaki, M. Bando and K. Matumoto, Phys. Rev. Lett. 56 (1986) 1335;

T. Akiba and T. Yanagida, Phys. Lett. B 169 (1986) 432;

T. Appelquist, D. Nash and L.C.R. Wijewardhana, Phys. Rev. Lett. 60 (1988) 2575;

V.A. Miransky, M. Tanabashi and K. Yamawaki, Mod. Phys. Lett. A 4 (1989) 1043; Phys. Lett. B 221 (1989) 177;

C.N. Leung, S.T. Love and W.A. Bardeen, Nucl. Phys. B 323 (1989) 493;

W.A. Bardeen, C.T. Hill and M. Lindner, Phys. Rev. D 41 (1990) 1647.

[3] J.B. Kogut, E. Dagotto and A. Kocic, Phys. Rev. Lett. 60 (1988) 772; Nucl. Phys. B 317 (1989) 253;

E. Dagotto, A. Kocic and J.B. Kogut, Nucl. Phys. B 331 (1990) 500.

[4] S.P. Booth, R.D. Kenway and B.J. Pendleton, Phys. Lett. B 228 (1989) 115.

[5] M. Salmhofer and E. Seiler, MPI preprint MPI-PAE/PTh$84 / 89$ (1989).

[6] S. Duane, A.D. Kennedy, B.J. Pendleton and D. Roweth, Phys. Lett. B 195 (1987) 216.

[7] M. Lüscher, DESY preprint DESY 90/005 (1990).

[8] M. Göckeler, R. Horseley, E. Laermann, P. Rakow, G. Schierholz, R. Sommer and U.-J. Wiese, in preparation.

[9] M. Lüscher, unpublished (1989).

[10] P.E.L. Rakow, DESY preprint DESY 90/029 (1990).

[11] C.N. Leung, S.T. Love and W.A. Bardeen, Nucl. Phys. B 273 ( 1986$) 649$.

[12] V.A. Miransky, Nuovo Cimento 90 A (1985) 149; Sov. Phys. JETP 61 (1985) 905;

P.I. Fomin, V.P. Gusynin, V.A. Miransky and Yu.A. Sitenko, Riv. Nuovo Cimento 6 (1983) 1. 